\title{
Average Polarization of Electromagnetic Gaussian Schell-Model Beams through Anisotropic Non-Kolmogorov Turbulence
}

\author{
Yuanhang ZHAO', Yixin $Z H A N G^{1,2}$, Qiu $W A N G^{1}$ \\ ${ }^{1}$ School of Science, Jiangnan University, Wuxi 214122, China \\ ${ }^{2}$ Jiangsu Provincial Research Center of Light Industrial Optoelectronic Engineering and Technology, Wuxi 214122, China \\ yuanhang133@sina.com,zyx@jiangnanedu.cn, 13589017702@163.com
}

Manuscript received April 24, 2016

\begin{abstract}
Polarization properties of electromagnetic Gaussian Schell-model beams propagating through the anisotropic non-Kolmogorov turbulence of marine-atmosphere channel are studied based on the cross-spectral density matrix. Detailed analysis shows that the average polarization decreases with increasing the spectral index, inner scale of turbulence and generalized refractive-index structure parameter. We find the effects of anisotropic turbulence on the average polarization is less than that of the isotropic turbulence and the depolarization effect of turbulence in marine-atmosphere is larger than terreneatmosphere. The electromagnetic Gaussian Schell-model beam with the parameters of smaller $\delta_{y y}, \delta_{x x}$ and $A_{x}$, but larger $A_{y}$ will reduce the interference of turbulence.
\end{abstract}

\section{Keywords}

Average polarization, electromagnetic Gaussian Schell-model beams, anisotropic non-Kolmogorov turbulence, marine-atmosphere

\section{Introduction}

Polarization is the important property to the light; it has a great number of applications both in the quantum and classical realms. Several authors have investigated the effects of turbulence and the partially coherent source on the polarization of electromagnetic Gaussian Schell-mode (EGSM) beams propagation in atmosphere [1-4]. Tang et al. propose a coherent multilevel polarization shift keying (MPOLSK) modulation scheme using the spatial diversity detection in a free-space optical (FSO) turbulence channel [5]. Wang et al. have derived analytical propagation formulae for the degree of cross-polarization (SDCP) of two Gaussian Schell-mode (GSM) beams propagation through non-Kolmogorov turbulence in the region of superposition [6]. Roychowdhury et al. find that the degree of polarization of the electromagnetic beam tends to the value that it has in the source plane [7]. Luo et al. have demonstrated how the electromagnetic spectral Gaussian Schell-model (EM SGSM) beams evolve in the atmospheric turbulence [8]. Recently, considerable efforts have been made to probe the effects of anisotropic turbulence on the optical wave propagation in atmosphere. Toselli et al. proposed power spectrum models of non-Kolmogorov turbulence to theoretically investigate the effect of anisotropy [9] and analyzed the impact of the spectral index variations on the long-term beam spread and scintillation index of the plane wave and spherical wave for several anisotropic coefficient values $\zeta[10]$ in the weak turbulence condition. The effect of anisotropic Kolmogorov turbulence on the log-amplitude correlation function for plane wave and spherical wave fields is investigated in [11], [12]. Yao et al. explored the effects of the anisotropic parameter on the spectral density, the spectral degree of coherence and on the spectral degree of polarization of the GSM beam [13]. A new closed-form expression for the mean square temporal width of Gaussian-beam-wave pulses passing horizontally through strong anisotropic atmospheric turbulence is developed by Chen et al. [14]. Cui et al. developed a theoretical consequence of the long-exposure turbulence modulation transfer function (MTF) for both plane and spherical waves propagating through anisotropic non-Kolmogorov turbulence [15].

Investigating marine atmospheric turbulence is necessary for the design of optical imaging system and optical communication system. Korotkova et al. providing a methodology for computing the probability density function of the laser beam intensity in the maritime environment using field measurements [16] and Cui et al. [17] derived a new analytic expression of the MTF to describe the degrading effects of marine atmospheric turbulence on an optical imaging system. The polarization fluctuation model of GSM beams propagating through the anisotropic non-Kolmogorov turbulence of marine/terrene-atmosphere was obtained under the restrain of turbulence with the Prandtl number $\operatorname{Pr}=0.72$ and the Obukhov-Corrsin coefficient $\beta=0.72$ in the narrow range of the spectral index of nonKolmogorov turbulence $3.47<\alpha<3.87$ [18]. But, to the best of our knowledge, there is no report on the polariza- 
tion fluctuations of EGSM beams propagating in anisotropic non-Kolmogorov turbulence of marine/terrene-atmosphere channel.

The aim of this paper is to develop a theoretical model for the average polarization fluctuations of EGSM beams in marine/terrene-atmosphere channel with anisotropic non-Kolmogorov turbulence. In Sec. 2 and 3 the model for the average polarization of GSM beams based on the cross-spectral density matrix is investigated in detail. Numerical results and discussions are given in Sec. 4. Conclusions are presented in Sec. 5.

\section{Cross-Spectral Density Matrix of EGSM Beams}

The second-order coherence and polarization properties of EGSM beams can be characterized by the $2 \times 2$ electric cross-spectral density matrix [19]

$$
\begin{array}{r}
\vec{W}\left(\mathbf{r}_{1}, \mathbf{r}_{2}, \omega\right)=\left[W_{l m}\left(\mathbf{r}_{1}, \mathbf{r}_{2}, \omega\right)\right]=\left\langle E_{l}^{*}\left(\mathbf{r}_{1}, \omega\right) E_{m}\left(\mathbf{r}_{2}, \omega\right)\right\rangle, \\
l=x, y, \quad m=x, y
\end{array}
$$

where $E_{l}\left(\mathbf{r}_{1}, \omega\right)$ and $E_{m}\left(\mathbf{r}_{2}, \omega\right)$ are the mutually orthogonal components of the electric field at point $\mathrm{P}\left(\mathbf{r}_{1}\right)$ and $\mathrm{P}\left(\mathbf{r}_{2}\right)$, representing a fluctuating of electric field. The asterisk denotes the complex conjugate and the angular brackets denote the average taken over a statistical ensemble of realizations of the electric field.

The components of EGSM beams in the turbulent marine-atmosphere can be written as [20]

$$
\begin{gathered}
E_{l, m}(\boldsymbol{\rho}, z, \omega)=-\frac{\mathrm{j} k}{2 \pi z} \exp (\mathrm{j} k z) \\
\iint E_{l, m}{ }^{(0)}\left(\boldsymbol{\rho}^{\prime}, \omega\right) \exp \left[\frac{\mathrm{j} k}{2 z}\left(\boldsymbol{\rho}-\boldsymbol{\rho}^{\prime}\right)^{2}\right] \times \exp \left[\psi\left(\boldsymbol{\rho}, \boldsymbol{\rho}^{\prime}, z, \omega\right)\right] d^{2} \boldsymbol{\rho}^{\prime}, \\
l=x, y, \quad m=x, y
\end{gathered}
$$

where $k=2 \pi / \lambda=\omega / c$ is the wave number, $\omega$ is the frequency, $z$ is the propagation distance, $\lambda$ is the wavelength and $\psi(\boldsymbol{\rho}, \boldsymbol{\rho} ', z, \omega)$ is the random part of the complex phase of a spherical wave propagating through the non-Kolmogorov turbulence of marine-atmosphere specified at any two points with position vectors $\boldsymbol{\rho}$ and $\boldsymbol{\rho}$ ' in the source plane.

Substituting (2) in (1), the cross-spectral density matrix elements of the electric field at the position $\mathbf{r}_{i}=\left(\boldsymbol{\rho}_{i}, z\right)$ in the output plane can be obtained [7].

$$
\begin{aligned}
& W_{l m}\left(\boldsymbol{\rho}_{1}, \boldsymbol{\rho}_{2}, z ; \omega\right) \\
& \quad=\left(\frac{k}{2 \pi z}\right)^{2} \iiint \int W_{l m}^{(0)}\left(\boldsymbol{\rho}_{1}^{\prime}, \boldsymbol{\rho}_{2}^{\prime}, 0 ; \omega\right) \\
& \quad \times \exp \left\{\frac{-\mathrm{j} k}{2 z}\left[\left(\boldsymbol{\rho}_{1}-\boldsymbol{\rho}_{1}^{\prime}\right)^{2}-\left(\boldsymbol{\rho}_{2}-\boldsymbol{\rho}_{2}^{\prime}\right)^{2}\right]\right\} \\
& \quad \times\left\langle\exp \left[\psi^{*}\left(\boldsymbol{\rho}_{1}, \boldsymbol{\rho}_{1}^{\prime}, z ; \omega\right)+\psi\left(\boldsymbol{\rho}_{2}, \boldsymbol{\rho}_{2}^{\prime}, z ; \omega\right)\right]\right\rangle_{a} d^{2} \boldsymbol{\rho}_{1}^{\prime} d^{2} \boldsymbol{\rho}_{2}^{\prime}
\end{aligned}
$$

Here $\left\langle\exp \left[\psi^{*}\left(\boldsymbol{\rho}_{1}, \boldsymbol{\rho}_{1}^{\prime}, z ; \omega\right)+\psi\left(\boldsymbol{\rho}_{2}, \boldsymbol{\rho}_{2}^{\prime}, z ; \omega\right)\right]\right\rangle_{a}$ is the phase correlation function and is given by [20]

$$
\begin{aligned}
& \left\langle\exp \left[\psi^{*}\left(\boldsymbol{\rho}_{1}, \boldsymbol{\rho}_{1}^{\prime}, z ; \omega\right)+\psi\left(\boldsymbol{\rho}_{2}, \boldsymbol{\rho}_{2}^{\prime}, z ; \omega\right)\right]\right\rangle_{a} \\
& =\exp \left[-4 \pi^{2} k^{2} z \int_{0}^{1} d \xi \int_{0}^{\infty} d \kappa \kappa \phi_{n}(\kappa)\right. \\
& \times\left[1-\mathrm{J}_{0}\left[\left|(1-\xi)\left(\boldsymbol{\rho}_{1}-\boldsymbol{\rho}_{2}\right)+\xi\left(\boldsymbol{\rho}_{1}^{\prime}-\boldsymbol{\rho}_{2}^{\prime}\right)\right| \kappa\right]\right]
\end{aligned}
$$

where $\mathrm{J}_{0}(\cdot)$ is the Bessel function of zero order.

In the paraxial optical channel, after approximating the Bessel function by its two first terms: $\mathrm{J}_{0}(x) \approx 1-x^{2} / 4$, the phase correlation function can be approximated as

$$
\begin{aligned}
& \left\langle\exp \left[\psi^{*}\left(\boldsymbol{\rho}_{1}, \boldsymbol{\rho}_{1}^{\prime}, z ; \omega\right)+\psi\left(\boldsymbol{\rho}_{2}, \boldsymbol{\rho}_{2}^{\prime}, z ; \omega\right)\right]\right\rangle_{a} \\
& \approx \exp \left\{-\frac{\pi^{2} k^{2} z}{3}\left[\left(\boldsymbol{\rho}_{1}-\boldsymbol{\rho}_{2}\right)^{2}+\left(\boldsymbol{\rho}_{1}-\boldsymbol{\rho}_{2}\right)\left(\boldsymbol{\rho}_{1}^{\prime}-\boldsymbol{\rho}_{2}^{\prime}\right)\right.\right. \\
& \left.\left.+\left(\boldsymbol{\rho}_{1}^{\prime}-\boldsymbol{\rho}_{2}^{\prime}\right)^{2}\right] \int_{0}^{\infty} d \kappa \kappa^{3} \phi_{n}(\kappa)\right\} \\
& \approx \exp \left\{-\frac{1}{\rho_{H}^{2}}\left[\left(\boldsymbol{\rho}_{1}-\boldsymbol{\rho}_{2}\right)^{2}+\left(\boldsymbol{\rho}_{1}-\boldsymbol{\rho}_{2}\right)\left(\boldsymbol{\rho}_{1}^{\prime}-\boldsymbol{\rho}_{2}^{\prime}\right)\right.\right. \\
& \left.\left.\quad+\left(\boldsymbol{\rho}_{1}^{\prime}-\boldsymbol{\rho}_{2}^{\prime}\right)^{2}\right]\right\}
\end{aligned}
$$

where $\rho_{\mathrm{H}}$ is the lateral coherence length of the spherical wave in marine/terrene-atmosphere channel and

$$
\rho_{H}^{2}(\omega)=\left[3.257 k^{2} z \int_{0}^{\infty} d \kappa \kappa^{3} \phi_{n}(\kappa)\right]^{-1}
$$

where $\phi_{n}(\kappa)$ is the power spectrum of refractive-index fluctuations of turbulence.

\section{Average Polarization of EGSM Beams}

In this paper we employ the anisotropic non-Kolmogorov power spectrum reported in [10], [11] for marine/terrene-atmosphere by using generalized von Karman model [10], [12], marine/terrene-atmosphere model [18], [20]. According to the discussion in [9], we apply the approximation of the anisotropy existing along the direction of propagation ( $\mathrm{z}$ axis) of the beam, and by the discussions in [21] for the spectral model of non-Kolmogorov atmospheric turbulence [20], the power spectrum of refractiveindex fluctuations of non-Kolmogorov turbulence can be expressed as [18]

$$
\begin{aligned}
\phi_{n}(\kappa)= & A(\alpha) C_{n}^{2} \zeta^{2}\left[1+a \frac{\kappa_{\zeta}}{\kappa_{H}}+b\left(\frac{\kappa_{\zeta}}{\kappa_{H}}\right)^{(6-\alpha) / 2}\right] \\
& \times \frac{\exp \left(-\kappa_{\zeta}^{2} / \kappa_{H}^{2}\right)}{\left(\kappa_{\zeta}^{2}+\kappa_{0}^{2}\right)^{\alpha / 2}}
\end{aligned}
$$

where $3<\alpha<5, \kappa^{2}=\left(\kappa_{x}^{2}+\kappa_{y}^{2}\right), \kappa_{\zeta}=\sqrt{\kappa_{z}^{2}+\zeta^{2} \kappa^{2}}, \zeta$ is 
an effective anisotropic factor, $\kappa_{0}=2 \pi / L_{0}, L_{0}$ is the outer scale of turbulence, $C_{n}{ }^{2}$ is the generalized refractive-index structure parameter with units $\mathrm{m}^{3-\alpha}, \kappa_{\mathrm{H}}=c(\alpha) / l_{0}, l_{0}$ is the inner scale of turbulence, and

$$
\begin{aligned}
c(\alpha)= & \left\{\pi A ( \alpha ) \left[\Gamma\left(\frac{3}{2}-\frac{\alpha}{2}\right)\left(\frac{3-\alpha}{3}\right)\right.\right. \\
& +a \Gamma\left(2-\frac{\alpha}{2}\right)\left(\frac{4-\alpha}{3}\right) \\
& \left.\left.+b \Gamma\left(3-\frac{3 \alpha}{4}\right)\left(\frac{4-\alpha}{2}\right)\right]\right\}^{1 /(\alpha-5)} .
\end{aligned}
$$

For Kolmogorov $\alpha=11 / 3$ and isotropic turbulence $\zeta=1$, Equation (6) is reduced to the power spectrum of refractive-index fluctuations of Kolmogorov turbulence of marine/terrene-atmosphere.

By the marine/terrene-atmosphere spectrum (6), the lateral coherence length of the spherical wave is given by

$$
\begin{aligned}
\rho_{H}^{-2}= & 3.257 k^{2} z A(\alpha) C_{n}^{2} \zeta^{-2} \int_{0}^{\infty}\left[\kappa_{\zeta}^{3}+a \frac{\kappa_{\zeta}^{4}}{\kappa_{H}}+b \frac{\kappa_{\zeta}^{6-\alpha / 2}}{\kappa_{H}^{3-\alpha / 2}}\right] \\
& \times \frac{\exp \left(-\kappa_{\zeta}^{2} / \kappa_{H}^{2}\right)}{\left(\kappa_{\zeta}^{2}+\kappa_{0}^{2}\right)^{\alpha / 2}} \mathrm{~d} \kappa_{\zeta} .
\end{aligned}
$$

Making use of the integral formula [20]

$$
\begin{aligned}
& \int_{0}^{\infty} d \kappa^{\prime} \kappa^{\prime 2 \mu} \frac{\exp \left(-\kappa^{\prime 2} / \kappa_{H}^{2}\right)}{\left(\kappa^{\prime 2}+\kappa_{0}^{2}\right)^{\alpha / 2}} \\
& =\frac{1}{2} \kappa_{0}^{2 \mu+1-\alpha} \Gamma\left(\mu+\frac{1}{2}\right) \mathrm{U}\left(\mu+\frac{1}{2} ; \mu-\frac{1}{3} ; \frac{\kappa_{0}^{2}}{\kappa_{H}^{2}}\right)
\end{aligned}
$$

we find that

$$
\begin{aligned}
\rho_{H}^{-2} & =1.629 k^{2} z A(\alpha) C_{n}^{2} \varsigma^{-2}\left[\kappa_{0}^{4-\alpha} \mathrm{U}\left(2 ; \frac{7}{6} ; \frac{\kappa_{0}^{2}}{\kappa_{H}^{2}}\right)\right. \\
& +\frac{a}{\kappa_{H}} \kappa_{0}^{5-\alpha} \mathrm{U}\left(\frac{5}{2} ; \frac{5}{3} ; \frac{\kappa_{0}^{2}}{\kappa_{H}^{2}}\right) \\
& \left.+\frac{b}{\kappa_{H}^{3-\alpha / 2}} \kappa_{0}^{7-3 \alpha / 2} \Gamma\left(\frac{14-\alpha}{4}\right) \mathrm{U}\left(\frac{14-\alpha}{4} ; \frac{32-3 \alpha}{12} ; \frac{\kappa_{0}^{2}}{\kappa_{H}^{2}}\right)\right]
\end{aligned}
$$

where $\mathrm{U}(a ; b ; z)$ is the confluent hypergeometric function of the second kind.

The elements of the electric cross-spectral density matrix can be expressed in the form [22]

$$
\begin{array}{r}
W_{l m}{ }^{(0)}\left(\boldsymbol{\rho}_{1}^{\prime}, \boldsymbol{\rho}_{2}^{\prime}, z ; \omega\right) \\
=\sqrt{S_{l}^{(0)}\left(\boldsymbol{\rho}_{1}^{\prime}, \omega\right)} \sqrt{S_{m}^{(0)}\left(\boldsymbol{\rho}_{2}^{\prime}, \omega\right)} \mu_{l m}^{(0)}\left(\boldsymbol{\rho}_{2}^{\prime}-\boldsymbol{\rho}_{1}^{\prime}, \omega\right) \\
(l, m=x, y)
\end{array}
$$

where $S_{l}^{(0)}\left(\boldsymbol{\rho}_{\mathbf{1}}, \omega\right)$ and $S_{m}{ }^{(0)}\left(\boldsymbol{\rho}_{\mathbf{2}}{ }^{\prime}, \omega\right)$ denote the spectral density of the component $E_{l}$ and $E_{m}$, and $\mu_{l m}^{(0)}\left(\boldsymbol{\rho}_{2}^{\prime}-\boldsymbol{\rho}_{1}^{\prime}, \omega\right)$ denotes the spectral degree of coherence of the field which is given by the expression [23]

$$
\begin{array}{r}
S_{l}^{(0)}\left(\boldsymbol{\rho}^{\prime}, \omega\right)=A_{l}^{2} \exp \left[-\boldsymbol{\rho}^{\prime 2} /\left(2 \sigma_{l}^{2}\right)\right], \\
\mu_{l m}^{(0)}\left(\boldsymbol{\rho}_{2}^{\prime}-\boldsymbol{\rho}_{1}^{\prime}, \omega\right)=B_{l m} \exp \left[-\left(\boldsymbol{\rho}_{2}^{\prime}-\boldsymbol{\rho}_{1}^{\prime}\right)^{2} /\left(2 \delta_{l m}^{2}\right)\right] \\
(l, m=x, y)
\end{array}
$$

where the coefficients $A_{l}, B_{l m}, \sigma_{l}$ and $\sigma_{l m}$ are independent of position but they generally depend on the frequency $\omega . \sigma_{l}$ is the width of the source beam and $\sigma_{l m}$ is the source correlation coefficient. Moreover, $B_{l m}$ is the complex correlation coefficient of the electric field and satisfy relations $B_{l m}=1$ $(l=m),\left|B_{l m}\right| \leq 1(l \neq m)$ and $B_{l m}=B^{*}{ }_{l m}$.

The cross-spectral density matrices of EGSM beams are given by expression in the form

$$
\begin{aligned}
W_{l m}{ }^{(0)}\left(\boldsymbol{\rho}_{1}^{\prime}, \boldsymbol{\rho}_{2}^{\prime}, z ; \omega\right) & =A_{l} A_{m} \exp \left[-\frac{\boldsymbol{\rho}_{1}^{\prime 2} \sigma_{m}^{2}+\boldsymbol{\rho}_{2}^{\prime 2} \sigma_{l}^{2}}{4 \sigma_{l}^{2} \sigma_{m}^{2}}\right] \\
& \times B_{l m} \exp \left[-\frac{\left(\boldsymbol{\rho}_{2}^{\prime}-\boldsymbol{\rho}_{1}^{\prime}\right)^{2}}{\left(2 \delta_{l m}^{2}\right)}\right] .
\end{aligned}
$$

Based on the discussion on the elements of the crossspectral density matrix of the electric field in the output plane [8] and after a straightforward calculation, we have the cross-spectral density matrix of the EGSM beam in the output plane $(\boldsymbol{\rho}, z)$

$$
\begin{aligned}
& W_{l m}\left(\boldsymbol{\rho}, \boldsymbol{\rho}^{\prime}, z ; \omega\right)= \\
& \quad \frac{A_{l} A_{m} B_{l m}}{\Delta_{l m}} \exp \left(-\frac{\boldsymbol{\rho}^{2}\left(\sigma_{l}^{2}+\sigma_{m}^{2}\right)+4 \sigma_{l}^{2} \sigma_{m}^{2} \gamma_{l m} \boldsymbol{\rho}^{\prime 2}}{4 \sigma_{i}^{2} \sigma_{j}^{2} \Delta_{i j}}\right) \\
& \quad \times \exp \left\{\left[\frac{\mathrm{i} k\left(\Delta_{l m}-1\right)}{z \Delta_{l m}}-\frac{1}{4 \Delta_{l m}}\left(\frac{1}{\sigma_{l}^{2}}-\frac{1}{\sigma_{m}^{2}}\right)\right] \boldsymbol{\rho} \boldsymbol{\rho}^{\prime}\right\} \\
& \text { where } \quad \Delta_{l m}=\left(\frac{z}{k}\right)^{2}\left[\left(\frac{1}{\sigma_{l}^{2}}+\frac{1}{\sigma_{m}^{2}}\right) \gamma_{l m}-\left(\frac{\sigma_{m}^{2}-\sigma_{l}^{2}}{4 \sigma_{l}^{2} \sigma_{m}^{2}}+\frac{\mathrm{i} k}{2}\right)^{2}\right] \quad \text { and } \\
& \gamma_{l m}=\frac{1}{16}\left(\frac{1}{\sigma_{l}^{2}}+\frac{1}{\sigma_{m}^{2}}\right)+\frac{1}{2 \delta_{l m}^{2}}+\frac{1}{\rho_{\mathrm{H}}^{2}} .
\end{aligned}
$$

The degree of polarization of the EGSM beam at the position $(\boldsymbol{\rho}, z)$ can be expressed as follows

$$
P(\rho, z, \lambda)=\sqrt{1-\frac{4 \operatorname{Det} W(\rho, z, \omega)}{[\operatorname{Tr} W(\rho, z, \omega)]^{2}}}
$$

where $\operatorname{Tr}$ is the trace of the cross-spectral density matrix. If the electric field components are uncorrelated, that is, $B_{x y}=B_{y x}=0$ [8], Equation (16) is simplified to

$$
P(\rho, z, \omega)=\left|\frac{W_{x x}(\rho, z ; \omega)-W_{y y}(\rho, z ; \omega)}{W_{x x}(\rho, z ; \omega)+W_{y y}(\rho, z ; \omega)}\right|
$$


where

$$
\begin{array}{r}
W_{l l}(\rho, z ; \omega)=\frac{\mathrm{A}_{l}^{2}}{\Delta_{l l}} \exp \left\{-\left[\frac{\left(1+2 \sigma_{l}^{2} \gamma_{l l}\right)}{2 \Delta_{l l} \sigma_{l}^{2}}-\frac{\mathrm{i} k\left(\Delta_{l l}-1\right)}{z \Delta_{l l}}\right] \rho^{2}\right\} \\
(l=x, y)
\end{array}
$$

The average cross-spectral density matrix elements in the receiving aperture are given by

$$
\begin{aligned}
\bar{W}_{l l}(\rho, z ; \omega)= & \frac{\pi \mathrm{A}_{l}^{2}}{\Delta_{l l}\left[\frac{\left(1+2 \sigma_{l}^{2} \gamma_{l l}\right)}{2 \Delta_{l l} \sigma_{l}^{2}}-\frac{\mathrm{i} k\left(\Delta_{l l}-1\right)}{z \Delta_{l l}}\right]} \\
& \times\left[\exp \left\{-\left[\frac{\left(1+2 \sigma_{l}^{2} \gamma_{l l}\right)}{2 \Delta_{l l} \sigma_{l}^{2}}-\frac{\mathrm{i} k\left(\Delta_{l l}-1\right)}{z \Delta_{l l}}\right]\left(\frac{D}{2}\right)^{2}\right\}-1\right]
\end{aligned}
$$

where $D$ is the diameter of receiving aperture.

The average polarization (AP) of the EGSM beam in the receiving aperture is given by

$$
P_{A V G}(\rho, z, \omega)=\left|\frac{\bar{W}_{x x}(\rho, z ; \omega)-\bar{W}_{y y}(\rho, z ; \omega)}{\bar{W}_{x x}(\rho, z ; \omega)+\bar{W}_{y y}(\rho, z ; \omega)}\right| .
$$

\section{Numerical Results and Analysis}

To analyze the effects of anisotropic factor, inner scale, propagation distance, refractive-index structure parameter, the outer scale, and spectral index of non-Kolmogorov turbulence on the AP of the propagation beams, we calculated the AP of the EGSM beam propagation along a marine/terrene-atmosphere turbulence channel by using (20). The results of the simulations are shown in Figs. 1-7. To simplify the subsequent analysis, here we take $\sigma_{x}=\sigma_{y}=0.02 \mathrm{~m}$.

To compare the effects of marine-and terrene-atmosphere turbulence on the AP, in Fig. 1 and Fig. 2, we give

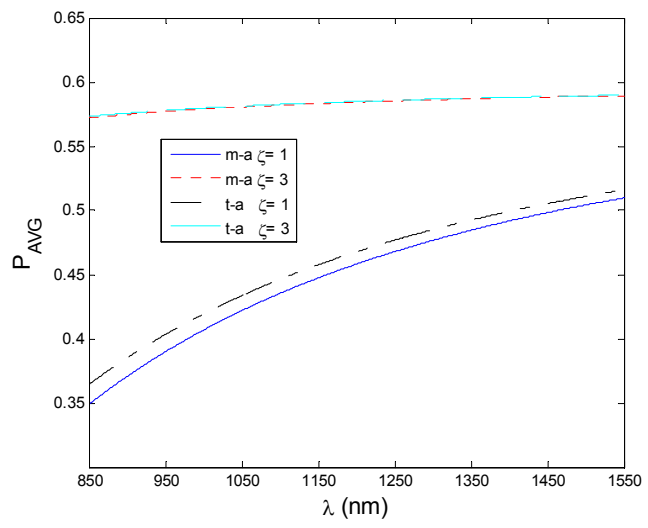

Fig. 1. AP as functions of wavelength $\lambda$ for marine/terrene atmosphere with different anisotropic factor $\zeta$. Here we take $\alpha=11 / 3$.

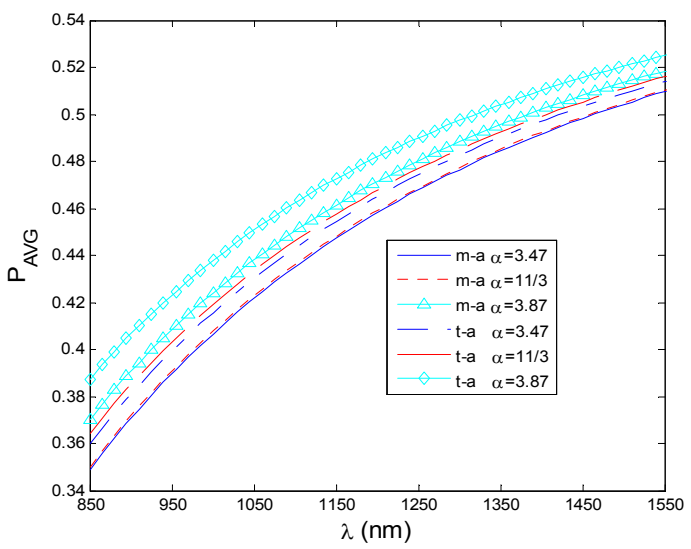

Fig. 2. $\mathrm{AP}$ as functions of wavelength $\lambda$ for marine/terrene atmosphere with different spectral index $\alpha$. Here we take $\zeta=1$

the curves of the AP for marine-and terrene-atmosphere turbulence links, where the curves depend that AP as function of wavelength $\lambda$ for different values of spectral index $\alpha$ (Fig. 1) and anisotropic factor $\zeta$ (Fig. 2) with parameters: $C_{n}{ }^{2}=10^{-14} \mathrm{~m}^{3-\alpha}, z=10 \mathrm{~km}, L_{0}=1 \mathrm{~m}$ and $l_{0}=1 \mathrm{~mm}$. One can see from the figures that the AP increases as wavelength and spectral index increases, and we know longwavelength infrared radiation possesses better all-weather transmission than the shorter wavelength radiation [22]. From Fig. 1, we can find the curve with larger anisotropic factor can lead to minor changes of AP compared with other curves, and the anisotropic turbulence will lead to lower polarization fluctuation.

In Fig. 3 and Fig. 4, we plot the curves of the AP as a function of the inner scale $l_{0}$ for given outer scale $L_{0}$ and refractive-index structure parameter $C_{n}{ }^{2}$ with parameters: $\alpha=11 / 3, z=10 \mathrm{~km}$, and $\lambda=1550 \mathrm{~nm}$. By Fig. 3 and Fig. 4 , it can be seen the smaller inner scale or refractiveindex structure parameter is, the stronger polarization fluctuation we get. At the same time, we can also find under the condition of same transport parameters, the polarization fluctuation in terrene-atmosphere channel is stronger than that in marine-atmosphere channel.

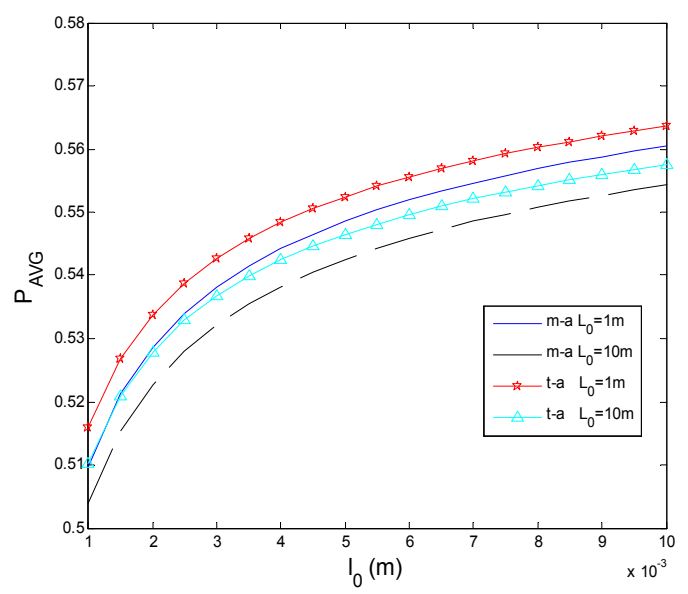

Fig. 3. AP as functions of inner scale $l_{0}$ for marine/ terrene atmosphere with different outer scale $L_{0}$. Here we take $C_{n}^{2}=10^{-14} \mathrm{~m}^{3-\alpha}$ 


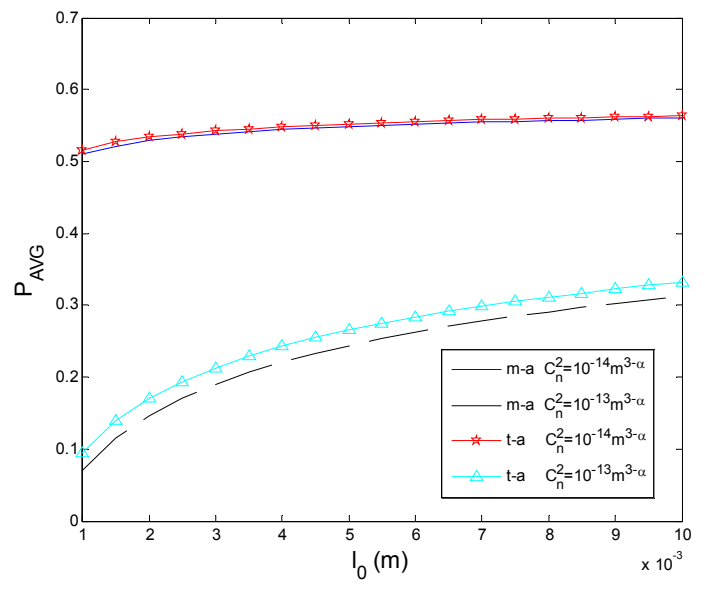

Fig. 4. AP as functions of inner scale $l_{0}$ for marine/terrene atmosphere with different refractive-index structure parameter $C_{n}{ }^{2}$. Here we take $L_{0}=1 \mathrm{~m}$.

We plot Fig. 5 to show the AP changes as the change of inner scale $l_{0}$ and propagation distance $z$ in marine-atmosphere channel. From this figure, it is clearly seen that the AP decreases as propagation distance $z$ increases, and from Figs. 3, 4 and 5, it can be seen as inner scale $l_{0}$ increases, the AP increases. This phenomenon displays that the turbulence with larger inner scale will have lower interference to polarization state of the EGSM beam propagating through marine atmosphere channel.

Figure 6 and 7 show the AP as functions of the outer scale $L_{0}$ and $A_{y}$ with the change of $\delta_{x x}, \delta_{y y}$ and $A_{x}$. From Fig. 6, we can find that the influence of outer scale on the AP can be ignored, and the AP decreases with the increasing of $\delta_{y y}$ and $\delta_{x x}$. Figure 7 shows that for the given $A_{y}$, the AP increases with decreasing $A_{x}$. But for given $A_{x}$, the AP has a least value as the $A_{y}$ increases from 0 to 5 or greater. Figure 6 and 7 indicate that if we transmit the EGSM beams with the smaller $\delta_{y y}, \delta_{x x}$ and $A_{x}$, but larger $A_{y}$, we will receive higher AP.

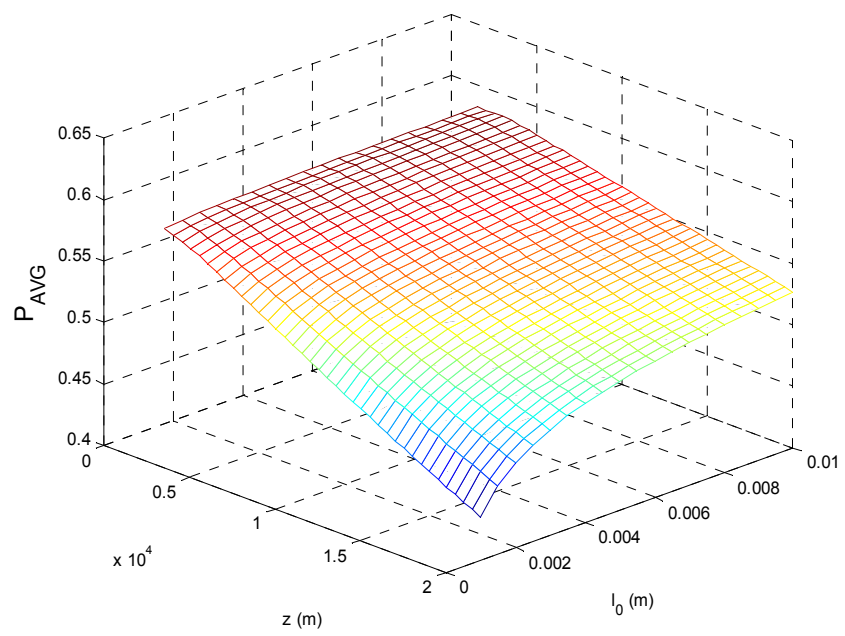

Fig. 5. AP as functions of propagation distance $z$ and inner scale $l_{0}$ in marine-atmosphere channel. Here we take $\lambda=1550 \mathrm{~nm}, \alpha=11 / 3, z=10 \mathrm{~km}$, and and $L_{0}=1 \mathrm{~m}$.

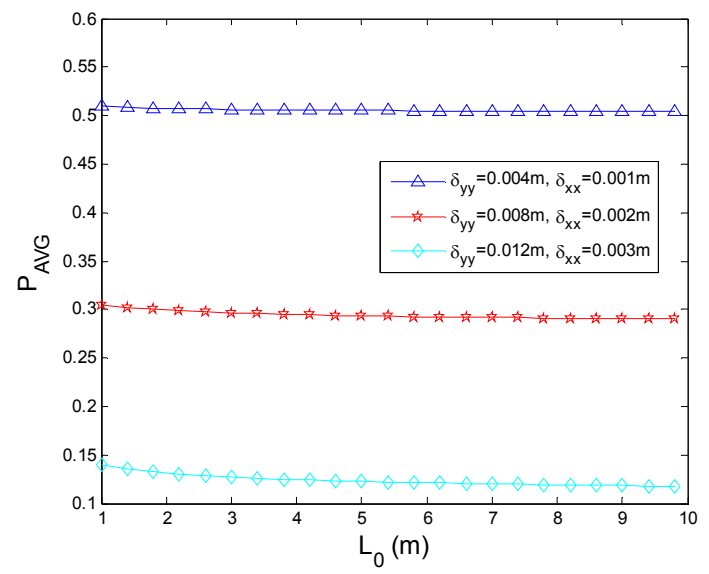

Fig. 6. AP as a function of outer scale $L_{0}$ in marineatmosphere channel with different $\delta_{x x}$ and $\delta_{y y}$.

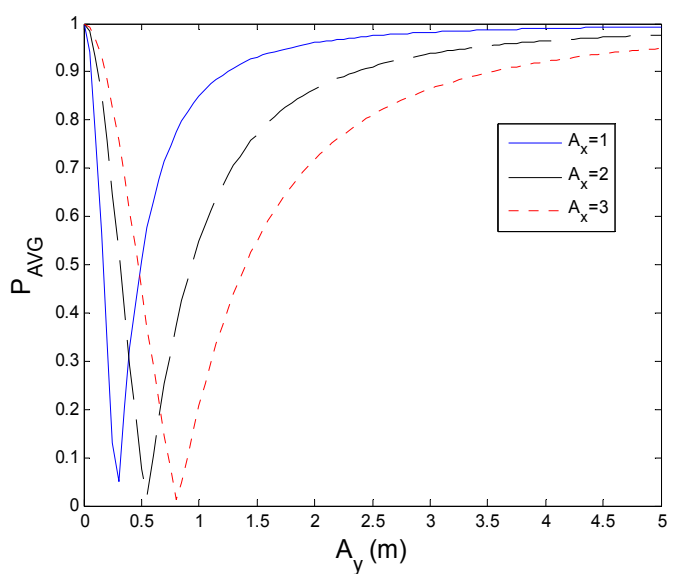

Fig. 7. $\mathrm{AP}$ as a function of $A_{y}$ in marine-atmosphere channel with different $A_{\mathrm{x}}$.

\section{Conclusion}

In this paper, the cross-polarization expression of EGSM beams propagating through the anisotropic nonKolmogorov turbulence of marine/terrene-atmosphere was obtained. The results show that the AP decreases with the decrease of inner scale and spectral index; the smaller scale eddies and anisotropic factor can lead to higher polarization fluctuation. We also find that the turbulence fade of the marine-atmosphere channel is larger than that of the terrene-atmosphere channels. The EGSM beam with the parameters of smaller $\delta_{y y}, \delta_{x x}$ and $A_{x}$, but larger $A_{z}$, will reduce the interference of turbulence. These results can help us choose and modulate the optimal parameters of EGSM beams to retain the robustness of polarization as the information carrier in quantum optical communications, and our result may be useful for remote sensing and optical communications. Due to the difference of refractive-index fluctuations caused by clean seawater turbulence and marine/terrene turbulence, in the future study, we will research the effects of turbulent water on the AP of EGSM beams. 


\section{Reference}

[1] KOROTKOVA, O., SALEM, M., WOLF, E. The far-zone behavior of the degree of polarization of electromagnetic beams propagating through atmospheric turbulence, Optics Communications, 2004, vol. 233, no. 4-6, p. 225-230. DOI: 10.1016/j.optcom.2004.01.005

[2] ZHU, Y., ZHAO, D. Propagation of a stochastic electromagnetic Gaussian Schell-model beam through an optical system in turbulent atmosphere. Applied Physics B, 2009, vol. 96. no. 1, p. 155-160. DOI: $10.1007 / \mathrm{s} 00340-009-3452-9$

[3] ZHU, S., CAI, Y., KOROTKOVA, O. Propagation factor of a stochastic electromagnetic Gaussian Schell-model beam. Optics Express, 2010, vol. 18, no. 12, p. 12587-12598. DOI: 10.1364/OE.18.012587

[4] DU, X., ZHAO, D. Polarization modulation of stochastic electromagnetic beams on propagation through the turbulent atmosphere. Optics Express, 2009, vol. 17, no. 6, p. 4257-4262. DOI: $10.1364 /$ OE.17.004257

[5] TANG, X., GHASSEMLOOY, Z., RAJBHANDARI, S., et al. Coherent heterodyne multilevel polarization shift keying with spatial diversity in a free-space optical turbulence channel. Journal of Lightwave Technology, 2012, vol. 30, no. 16, p. 2689-2695. DOI: $10.1109 /$ JLT.2012.2204859

[6] WANG, Y., SI, C., ZHANG, Y., et al. Cross-polarization properties of two Gaussian Schell-model beams through nonKolmogorov turbulence. Optics \& Lasers in Engineering, 2011, vol. 49, no. 11 , p. 1060-1064. DOI: 10.1016 /j.optlaseng. 2011.03 .016

[7] ROYCHOWDGHURY, H., PONOMARENKO, S. A., WOLF, E Change in the polarization of partially coherent electromagnetic beams propagating through the turbulent atmosphere. Journal of Modern Optics, vol. 52, no. 11, 2005, p. 1611-1618. DOI: $10.1080 / 09500340500064841$

[8] LUO, M., ZHAO, D. Propagation of electromagnetic spectral Gaussian Schell-model beams in atmosphere. Optics Communications, 2015, vol. 336, p. 98-102. DOI: 10.1016/j.optcom.2014.10.002

[9] TOSELli, I., AGRAWAL, B., RESTAINO, S. Light propagation through anisotropic turbulence. Journal of the Optical Society of America A, 2011, vol. 28, no. 3, p. 483-488. DOI: 10.1364/JOSAA.28.000483

[10] TOSELLI, I. Introducing the concept of anisotropy at different scales for modeling optical turbulence. Journal of the Optical Society of America A, 2014, vol. 31, no. 8, p. 1868-1875. DOI: 10.1364/JOSAA.31.001868

[11] GUDIMETLA, V. S. R., HOLMES, R. B., SMITH, C., NEEDHAM, G. Analytical expressions for the log-amplitude correlation function of a plane wave through anisotropic atmospheric refractive turbulence. Journal of the Optical Society of America A, 2012, vol. 29, no. 5, p. 832-840. DOI: 10.1364/JOSAA.29.000832

[12] GUDIMETLA, V. S. R., HOLMES, R. B., RIKER, J. F. Analytical expressions for the log-amplitude correlation function for spherical wave propagation through anisotropic non-Kolmogorov atmosphere. Journal of the Optical Society of America A, 2014, vol. 31, no. 1 , p. $148-154$. DOI: $10.1364 /$ JOSAA.31.000148

[13] YAO, M., TOSELli, I., KOROTKOVA, O. Propagation of electromagnetic stochastic beams in anisotropic turbulence. Optics Express, 2014, vol. 22, no. 26, p. 31608-31618. DOI: 10.1364/OE.22.031608

[14] CHEN, C., YANG, H., TONG, S., REN, B., LI, Y. Characterization of temporal pulse broadening for horizontal propagation in strong anisotropic atmospheric turbulence. Optics Express, 2015, vol. 23, no. 4, p. 4814-4828. DOI: 10.1364/OE.23.004814

[15] CUI, L., XUE, B., CAO, X., ZHOU, F. Atmospheric turbulence MTF for optical waves propagating through anisotropic nonKolmogorov atmospheric turbulence. Optics \& Laser Technology, 2014, vol. 63, p. 70-75. DOI: 10.1016/j.optlastec.2014.03.011

[16] KOROTKOVA, O., AVRAMOV-ZAMUROVIC, S., MALEKMADANI, R., et al. Probability density function of the intensity of a laser beam propagating in the maritime environment. Optics Express, 2011, vol. 19, no. 21, p. 20322-20331. DOI: 10.1364/OE.19.020322

[17] CUI, L., XUE, B., ZHOU, F. Atmospheric turbulence MTF for infrared optical waves' propagation through marine atmospheric turbulence. Infrared Physics \& Technology, 2014, vol. 65, p. 24-29. DOI: 10.1016/j.infrared.2014.03.004

[18] ZHAO, Y., ZHANG, Y., HU, Z., et al. Polarization of quantization Gaussian Schell-beams through anisotropic non-Kolmogorov turbulence of marine-atmosphere. Optics Communications, 2016, vol. 371, p. 178-183. DOI: 10.1016/j.optcom.2016.03.068

[19] WOLF, E. Unified theory of coherence and polarization of random electromagnetic beams. Physics Letters A, 2003, vol. 312, no. 5-6, p. 263-267. DOI: 10.1016/S0375-9601(03)00684-4

[20] ANDREWS, L. C., PHILLIPS, R. L. Laser Beam Propagation through Random Media. 2nd ed. New York: SPIE, 2005. ISBN: 9780819459480

[21] XUE, B., CUI, L., XUE, W., et al. Generalized modified atmospheric spectral model for optical wave propagating through non-Kolmogorov turbulence. Journal of the Optical Society of America A, vol. 28, no. 5, 2011, p. 912-916. DOI: 10.1364/ JOSAA.28.000912

[22] ROYCHOWDHURY, H., WOLF, E. Determination of the electric cross-spectral density matrix of a random electromagnetic beam. Optics Communications, 2003, vol. 226, p. 57-60. DOI: 10.1016/ j.optcom.2003.07.054

[23] KOROTKOVA, O., WOLF, E. Changes in the state of polarization of a random electromagnetic beam on propagation. Optics Communications, 2005, vol. 246, p. 35-43. DOI: 10.1016/ j.optcom.2004.10.078

\section{About the Authors ...}

Yuanhang ZHAO was born in Shandong province, China. He received the B.S. degree in 2009. He is currently pursuing the M.E. degree in the Jiangnan University. His research interest is polarization optics in telecommunications.

Yixin ZHANG (corresponding author) was born in Jiangsu province, China. He received the Ph. D degree in Nanjing University of Science and Technology, and has been a professor of Jiangnan University since 1994. He has authored and coauthored more than 200 scientific papers in major journals and international conferences. His research interest is wireless optics communication.

Qiu WANG was born in Jiangsu province, China. She received the B.S. degree in 2011. Her current research interest is beam spread. 